\title{
COMUNICACIÓN
}

\section{Presencia de Trypanosoma minasense (Kinetoplastida: Trypanosomatidae) en Alouatta palliata (Primates: Cebidae) de Costa Rica}

\author{
MISAEL CHINCHILLA*****, ADRIANA TROYO*, OLGA M. GUERRERO*, \\ GUSTAVO A. GUTIÉRREZ-ESPELETA** y RONALD SÁNCHEZ*
}

\section{Trypanosoma minasense (KINETOPLASTIDA: TRYPANOSOMATIDAE) IN Alouatta palliata (PRIMATES: CEBIDAE) OF COSTA RICA}

A Trypanosoma from the howler monkey Alouatta palliata was studied. Measures, as well as its biological characteristics (mice infection and failure to infect Triatominae) identify this parasite as Trypanosoma minasense. This organism is found for the first time in Costa Rica.

Key words: Trypanosomatidae, Trypanosoma minasense, Alouatta palliata, howler monkey.

\section{INTRODUCCIÓN}

Trypanosoma minasense fue reportado y descrito por primera vez por Carlos Chagas $^{1}$ parasitando un mono de la especie Hapalle penicillata, posteriormente clasificado como Callithrix penicillata, así como en otras especies de primates, a la par del Trypanosoma cruzi que luego estudió en detalle. Carlos Chagas menciona que $T$. minasense no produce patología importante para los animales mientras que T. cruzi produce la tripanosomiasis americana que hoy lleva su nombre.

La descripción original es sumamente simple pero ya se deduce que este tripanosomátido pertenece al subgénero Megatrypanum ${ }^{2}$, que en efecto no es patógeno para su hospedero.

Posteriormente, se informa el hallazgo de una infección del $19 \%$ en primates importados de Perú y Colombia pertenecientes al mismo género en el que se describió el parásito y a la especie Saimiri sciureus ${ }^{3}$. Más recientemente se hace una descripción morfológica un poco más detallada de la forma de tripomastigoto de este parásito $^{4}$. Un estudio morfogenético de $T$. minasense fue realizado también comparándolo con otros tripanosomas similares ${ }^{5}$. Otros investigadores cultivan estos parásitos en medio NNN suplementado con $20 \%$ de suero fetal bovino, realizando estudios morfogénicos de las formas de cultivo ${ }^{6}$. En otro estudio sobre 165 monos ardilla (S. sciureus y $S$. ustus) se encontró

* Centro de Investigación en Enfermedades Tropicales (CIET), Departamento de Parasitología, Facultad de Microbiología Universidad de Costa Rica.

** Escuela de Biología, Universidad de Costa Rica.

*** Escuela de Biología, Centro Regional de Occidente, Universidad de Costa Rica.

**** Departamento de Investigación, Universidad de Ciencias Médicas.

Dirección: Misael Chinchilla. Facultad de Microbiología, Universidad de Costa Rica. San José, Costa Rica, América Central. 
T. minasense en un $33,3 \%$ de esos animales. También se ha encontrado este parásito en monos de la Amazonia en Brasil ${ }^{8}$.

En Costa Rica, existen estudios sobre parásitos de Alouatta palliata ${ }^{9}$, pero este tripanosomátido no ha sido reportado. En esta comunicación informamos por primera vez de su presencia en un ejemplar de A. palliata capturado a raíz de un estudio integral y multidisciplinario que se hace en primates de Costa Rica y en el que participan diversos grupos de varias Universidades de Costa Rica.

\section{MATERIAL Y MÉTODOS}

Para realizar este estudio, los animales fueron capturados anestesiándolos con Ketamina y Xilosina (0,2 mg/kilo de peso), fueron sangrados de la arteria femoral, recogiendo la sangre en tubos al vacío con anticoagulante y cultivando ésta en medio de Rugai para el aislamiento de los parásitos. Al mismo tiempo, se hicieron extensiones sanguíneas directas, las cuales fueron fijadas con alcohol metílico y teñidas por el método convencional de Giemsa. Los hemocultivos fueron revisados a los 3 días, en los que se observaron las primeras formas desplazándose activamente. Después de los 10 días, se observó un crecimiento abundante, por lo que se hicieron preparaciones para teñirlas posteriormente $\mathrm{y}$ realizar el estudio del caso. Parte del cultivo fue usado para inocular ratones $\mathrm{C}_{3} \mathrm{H}$ vía intraperitoneal, los animales fueron revisados diariamente por examen directo y por concentración bajo el sistema de centrifugación para hematocrito.

\section{RESULTADOS}

Al estudiar las formas de epimastigoto del cultivo se observó que de acuerdo con su movimiento y sus características morfológicas eran similares a las de los subgéneros Herpetosoma y Megatrypanum. Después de 10 a 12 días de inoculados, los ratones presentaron pocas formas de tripomastigoto que al teñirlas y proceder a realizar las medidas de 30 ejemplares, indicaron los resultados que se indican en la Tabla 1 y cuya morfología se muestra en la Figura 1.

\section{DISCUSIÓN}

Las formas estudiadas resultaron ser similares

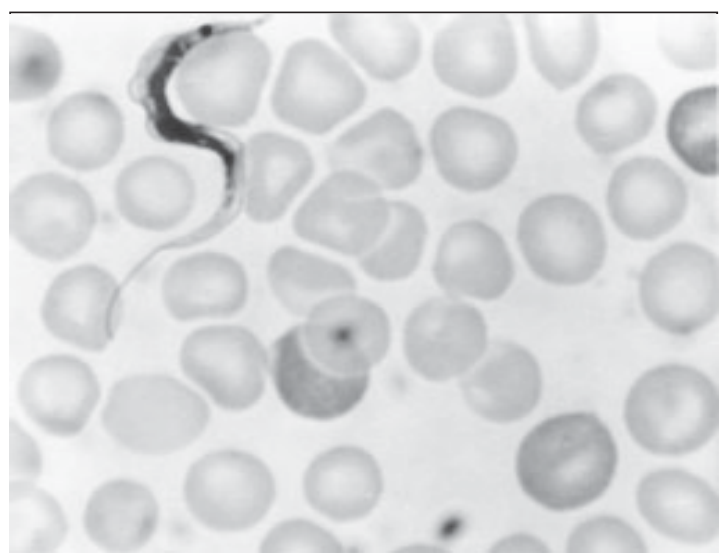

Figura 1. Formas del Trypanosoma minasense aisladas en ratón $\mathrm{C}_{3} \mathrm{H}$.

Tabla 1. Análisis comparativo de las medidas $(\mu \mathrm{m})$ del Trypanosoma encontrado y las de Trypanosoma diasi y Trypanosoma minasense*

\begin{tabular}{|c|c|c|c|c|c|c|c|c|c|c|}
\hline \multirow{2}{*}{$\begin{array}{c}\text { Medidas } \\
(\mu \mathrm{m})\end{array}$} & \multicolumn{4}{|c|}{ S Trypanosoma sp. (en estudio) $\mathbf{n}=\mathbf{3 0}$} & \multicolumn{3}{|c|}{ T. diasi $\mathrm{n}=4$} & \multicolumn{3}{|c|}{$T$. minasense $\mathbf{n}=\mathbf{3 9}$} \\
\hline & Media** & $\mathbf{s}$ & Max & Min & Media & Max & Min & Media & Max & Min \\
\hline $\mathrm{L}$ & 35,02 & 1,19 & 37,28 & 32,36 & 35,2 & 36,2 & 33,5 & 30,6 & 39 & 23 \\
\hline PK & 8,03 & 0,78 & 9,27 & 7,21 & 8,9 & 11,3 & 7,3 & 10,7 & 14 & 6,8 \\
\hline $\mathrm{KN}$ & 9,24 & 0,74 & 10,3 & 7,21 & 7,2 & 7,5 & 6,7 & 8,7 & 13,6 & 5,6 \\
\hline NA & 9,61 & 0,74 & 11,2 & 8,2 & 15,7 & 17,8 & 14 & 8,4 & 11,2 & 5,6 \\
\hline $\mathrm{F}$ & 8,14 & 0,95 & 10,3 & 7,21 & 7,8 & 8,6 & 7 & 6,6 & 9 & 5,4 \\
\hline
\end{tabular}

F: flagelo libre, KN: kinetoplasto a núcleo. L: largo total, NA: núcleo a extremo anterior PK: extremo posterior a kinetoplasto.

* Deane y Damasceno, 1961

** Max: Máximo, Min: mínimo, n: número de ejemplares, s: desviación estándar 
Trypanosoma minasense en Alouatta palliata (Primates: Cebidae) - M. Chinchilla et al.

a las informadas ${ }^{3,4,10}$ para $T$. minasense (Tabla 1 , Figura 1). Con la única especie que se podría confundir morfológicamente este organismo es con Trypanosoma diasi pero este último infecta fácilmente diversas especies de la familia Reduviidae y no infecta ratones ${ }^{10}$. En el presente estudio, las inoculaciones en ratones fueron exitosas. Al realizar xenodiagnósticos de los roedores positivos por el tripanosomátido los triatominos no se infectaron.

Otro tripanosomátido encontrado en el mono aullador es el T. lambrechti ${ }^{11}$ pero las medidas de este parásito difieren mucho, ya que su tamaño es mayor a la especie descrita en este informe.

Se concluye entonces, con base en los estudios anteriormente mencionados, que el parásito encontrado en el mono congo ( $A$. palliata) es $T$, (Megatrypanum) minasense, constituyendo éste el primer reporte en Costa Rica y probablemente en Centroamérica ya que no se han encontrado informes de este parásito en monos del género Alouatta. La referencia ${ }^{9}$ en que se menciona la presencia de $T$. minasense en Alouatta belzebul, es incorrecta pues en la cita original $^{10}$ a la que se hace referencia, no se menciona tal infección.

Estudios detallados sobre la biología, inmunología y genética de este parásito están en progreso.

\section{RESUMEN}

Se informa de la presencia de un tripanosomátido del mono aullador Alouatta palliata cuyas medidas, su capacidad de infectar ratones blancos y $\mathrm{C}_{3} \mathrm{H}$, y la no infección de Triatominos, lo clasifican como Trypanosoma minasense. Esta es la primera vez que se describe este parásito en monos de Costa Rica.

\section{REFERENCIAS}

1.- CHAGAS C. Trypanosoma minasense: Nota preliminar. Brazil-Medico 1908; 22: 471.

2.- HOARE C A. The classification of mammalian trypanosomes. Ergebn. Mikrobiol 1966; 39: 43-57.

3.- DUNN F L, LAMBRECHT F L, DU PLESSIS R. Trypanosomes of South American monkeys and marmosets. Am J Trop Med Hyg 1963; 12: 524-34.

4.- GONZALO A, TANTALEÁN M. Parasitic protozoa in neotropical primates. Laboratory Primate Newsletter 1996; 35: 1-19.

5.- ZICCARDI M, LAOURENÇO-DE-OLIVEIRA R. Morphological features of trypanosomes from squirrel monkeys from Brazilian Amazon. Mem Inst Oswaldo Cruz 1998; 93: 45-55.

6.- ZICCARDI M, LOWENÇO-DE-OLIVEIRA R, NOGUEIRA R. The haemoculture of Trypanosoma minasense Chagas, 1908. Mem Inst Oswaldo Cruz 1996; 91: 501-5.

7.- ZICCARDI M, LOURENÇO-DE-OLIVEIRA R. The infection rates of trypanosomes in squired monkeys at two sites in the Brazilian Amazon. Mem Inst Oswaldo Cruz 1997; 92: 465-70.

8.- ZICCARDI M, LOURENÇO-DE-OLIVEIRA R, LAINSON R, et al. Trypanosomes of non-human primates from the National Centre of Primates, Ananindeua, state of Para, Brazil. Mem Inst Oswaldo Cruz 2000; 95: 157-9.

9.- STUART M D, PENDERGAST V, RUMFELT S. Parasites of wild howlers (Alouatta spp). Int J Primat 1998; 19: 493-512.

10.- DEANE L M, DAMASCENO R G. Tripanosomídeos de mamíferos da regiào amazónica. II. Tripanosomas de macacos da Zona do Salgado, Estado do Pará. Rev Inst Med Trop Sào Paulo 1961; 3: 61-70.

11.- Deane L M, Batista D, Ferreira J A, de Souza H. Trypanosomideos de mamíferos da Regiáo Amazónica. V. Tripanosoma lambrechti Marinbell, 1968, en macacos do estado do amazonas. Brasil. Rev Inst Med Trop Sào Paulo 1970; 12: 1-7.

Agradecimientos: Este trabajo fue financiado en parte por la Vicerrectoría de Investigación de la Universidad de Costa Rica a través de los proyectos VI-III-A1-015 y 803-A1745 y el Departamento de Investigación de la Universidad de Ciencias Médicas. 